\title{
Exploring salient dimensions in a free sorting task: a cross-country study on elderly
}

populations

${ }^{* 1}$ Cliceri Danny, ${ }^{1}$ Dinnella Caterina, ${ }^{2}$ Depezay Laurence, ${ }^{2}$ Morizet David, ${ }^{3}$ Giboreau Agnés, ${ }^{4}$ Appleton

Katherine M., ${ }^{5}$ Hartwell Heather and ${ }^{2}$ Monteleone Erminio

${ }^{1}$ Department of Management of Agricultural, Food and Forestry Systems, University of Florence, Italy

${ }^{2}$ Sensory \& consumer science, Bonduelle corporate research, France

${ }^{3}$ Institute Paul Bocuse Research Centre, France

${ }^{4}$ Research Centre for Behaviour Change, Department of Psychology, Bournemouth University, UK

${ }^{5}$ The Foodservice and Applied Nutrition Research Group, Bournemouth University, UK

*Corresponding author: danny.cliceri@unifi.it

\section{Abstract}

Free sorting tasks have been widely applied on different age segments to study the categorization of foods. However the method has received little attention in the investigation of older adults' perception of foods. Given the importance of understanding elderly perceptions in order to develop acceptable products, the aim of this study was to investigate the suitability of a free sorting task on different age groups of healthy elderly consumers. The role of sensory and hedonic dimensions, beside the role of familiarity, was considered to better understand the process of food categorization. A free sorting and a liking task were applied on French and Italian elderly to study perception and preference of familiar (peas) and unfamiliar (sweetcorn) vegetables. Similarities between the categorization maps, the preference maps and the sensory maps from vegetable samples were assessed through the RV coefficient and map visual inspection.

The free sorting task was found to be a suitable method to use with healthy older adults, that allowed the detection of differences in the categorization of stimuli even among the more aged representatives of the elderly population. Familiarity with the product was the main factor affecting the categorization maps. Categorization maps from the familiar vegetable were found to be reliable to obtain information on sensory and hedonic dimensions, while maps obtained from the unfamiliar vegetable mainly depicted sensory variability.

Key words: free sorting task, older adults, familiarity, descriptive analysis, liking, vegetables

\section{Introduction}

Population aging represents one the most important global demographic trends of this century, considering that in 2050 one person in three will be elderly (United Nations, 2015). Besides population ageing, a further aspect that has to be considered is healthy life expectancy, namely the measure of years that a person is expected to live without disability. Investigations that involve indices of the healthy

41 life expectancy, such as DALYs (GBD 2013 DALYs and HALE Collaborators, 2015) or HLY (Robine \& 42 Camboise, 2013), typically show that this value stays constant despite an increase in the general life 
in a condition of activity limitation. In order to maintain high levels of health during the lifespan and avoid an excessive burden on health and care services, it is therefore vital to adopt strategies to increase healthy life expectancy. From the individual point of view, one way to promote a healthy life is undoubtedly to have a balanced diet that satisfies the nutritional requirements of the age segment. Aging is associated with an augmented risk of malnutrition (Hickson, 2006), which can lead to sarcopenia (Cruz-Jentoft et al., 2010) and subsequent frailty and dependency (Roubenoff, 2000). To prevent this negative spiral of inadequate food intake, malnutrition and the onset of disease, in the last years scholars have called for solutions to prevent malnutrition in older adults through the development of foods and modalities of consumption that consider the needs and preferences of the elderly population (Giacalone et al., 2016; Nyberg et al., 2015; Appleton et al. 2016).

The study of elderly consumers requires investigative tools that allow evaluation of the perceptions and preferences of this segment of the population in an effective and reliable way, while the majority of the methods used to study consumers' responses were developed with adults, without taking into account the physical and cognitive difficulties that may be present in elderly subjects. In healthy older adults most sensory and consumer methods can be applied (Methven et al., 2016). However the use of consumer tests with this segment of population should be evaluated carefully, due to the possible presence of difficulties related to the comprehension and use of rating scales (Dermiki et al., 2013), difficulties in the use of introspection processes, and a general tendency to have cognitive and perceptive fatigue with long and complex methodologies (Methven et al., 2016). Discriminant methods, such as ranking or paired tests, are typically the simplest methods to use with older adults (Barylko-Pikieln et al., 2004; Dermiki et al., 2014), although potential limitations include the lack of a direct indication of acceptability and the need for sufficient time when a high number of samples have to be assessed. A methodology with big potential, yet to be fully explored with older adults is the free sorting task (FST). The free sorting task is a method based on categorization, a natural cognitive process where objects with common characteristics are grouped and inference is made about their properties, in order to obtain considerable information with minimum cognitive effort (Rosch \& Lloyd, 1978). The method has been shown to be easily applicable with consumers considering that little training is required, quantitative rating systems are not requested, and in general the method is based on a simple and spontaneous cognitive process. In FST, subjects are provided with a varied number of samples and asked to evaluate and group them on the basis of their subjective criteria. Research involving FST on food products has highlighted the importance of the sensory dimension as a categorization criteria, and demonstrated that the maps from FST are often found to be highly correlated with the sensory maps obtained with descriptive analysis (DA) (Cartier et al., 2006). A further dimension relevant in food product categorization is the hedonic one (Ballester et al., 2008; Chollet $\&$ Valentin, 2000), even if only a limited effect on the structuring of similarity space is reported. Moreover different studies have highlighted a role for familiarity in foods categorization, where subjects with previous experience with the tested products tend to use higher-level types of categorization such as those based on the extrinsic properties of food (Solomon, 1997; Ballester et al., 2008). The role of familiarity in the categorization of food products has emerged also in cross-cultural studies, where cultures with different levels of familiarity with the tested products provide different spatial representations of them (Chrea et al., 2004; Blacher et al., 2007). In the domain of consumer research, FST has been used with children (Morizet et al., 2012; Varela \& Salvador, 2014), adolescents (Bucher et al., 2016) and adult respondents (Lawless et al., 1995; Lelièvre et al., 2009; Santosa et al., 2010; Nestrud \& Lawless, 2010; Deegan et al., 2010). The only study, of 
which we are aware, that has investigated the use of FST with food samples in older adults was carried out by Withers and colleagues (Withers et al., 2014). In this research, a variation of the basic sorting task, called Taxonomic free sorting, was coupled with hedonic liking ratings to produce an external preference map from consumer data. The study demonstrated the applicability of sorting methodologies with healthy older adults in general. However, the authors did not explore the categorization performance of different age segments of the elderly population, while the elderly population, despite often being considered as a single group, contains subjects that may differ considerably in perceptual abilities (Song et al., 2016) and in their familiarity with and liking for different food products (Mingioni et al., 2016). Hence, the variability within older adults may affect the main dimensions driving the categorization of food products.

In order to evaluate the performance of FST methodology within the elderly population, the main objective of this study was therefore to evaluate the suitability of FST in different age groups of healthy older adults. A further objective was to investigate the factors that were able to affect the categorization of samples in each considered segment. The influence of the sensory dimension on the process of categorization was assessed by comparing the categorization map obtained from FST against the sensory map from DA, while the influence of the hedonic dimension was assessed by comparing the categorization map against the preference map obtained from a liking task with the same subjects. Moreover, the study was carried out on a familiar and a unfamiliar product and in two different food cultures, that is the French and Italian one, to investigate the role of the experience of consumption on the creation of mental categories. Considering the importance of promoting the intake of healthy foods, the present study was conducted using vegetable products. In order to explore an approach where healthy food consumption is increased through the optimization of healthy foods already present in the diet of older adults (Appleton, 2016), the study was carried out using specific typologies of vegetables, which were pea, representative of the familiar product, and sweetcorn, representative of the unfamiliar product.

\section{Material and Methods}

\subsection{Products and Samples}

Pea and sweetcorn were selected as vegetable typologies because of their differential adoption in European food culture, where sweetcorn was introduced only in the second part of the $20^{\text {th }}$ century while pea has been present for several centuries (Pelt, 1993). Canned versions of peas and sweetcorn were chosen because of their large availability in the markets of the countries involved in the study and because they represent a convenient way to promote vegetable intake (Kapica \& Weiss, 2012). Ten canned pea (codes: $A, B, D, E, F, J, L, O, P, Q$ ) and eight canned sweetcorn (codes: $H, R, S, T, U, V, W, Z$ ) samples were considered for the study. The amount of each sample needed for the whole study was purchased from the producer company and from the same production batch, then delivered to the Institutions participating in the study. The samples were selected in order to cover as much as possible of the sensory spaces of peas and sweetcorn (i.e. diversity of size, texture, colour, flavour) and DA (Lawless \& Heymann, 2010) was carried out in order to confirm and quantify the sensory variability of samples. 
mean age 29.8 years, were selected for the DA of the pea samples. Eleven participants, 4 males and 7 females, mean age 30.1 years, were selected for the DA of the sweetcorn samples. After sample familiarization and sensory descriptor elicitation, the calibration and performance evaluation of each panel was assessed in three sessions where four samples were presented. Data were analyzed using Panel Check software (ver 1.4.0, Nofima, Tromso, Norway). Panel calibration was assessed using the multi-block PCA (Tucker-1), while assessor performance was assessed using the p*MSE plot. (Næs et al., 2010). Having completed the training, and after performance validation, panels participated in three evaluation sessions. In each session, ten samples of peas or eight samples of sweetcorn were evaluated in two sub-sets. Samples $(25 \mathrm{gr}$ ) were presented in a $100 \mathrm{cc}$ plastic cup identified by a 3-digit code. Samples presentation was balanced across participants. Pea samples were evaluated at $54-56^{\circ} \mathrm{C}$, while sweetcorn samples were evaluated at room temperature. Evaluations were performed in individual booths under white light for appearance description and under red light for the rest of the attributes. Data were collected with the software Fizz (ver.2.47.B, Biosystemes, Couternon, France). Sample differences for each attribute were assessed by a three way ANOVA mixed model using assessor and replicate as random factors, while sample was the fixed factor. Differences and similarities in sensory properties among samples were evaluated on a score plot and a correlation loading plot obtained from a Principal Component Analysis (PCA). PCA models were computed on panel averages of each significant sensory attribute $(p<0.05)$ arising from the ANOVA models. Data were analysed with the software Fizz (ver.2.47.B, Biosystemes, Couternon, France). The ANOVA model computed on DA data for the pea samples showed a significant sample effect for 23 of the 26 attributes. The first two components of the score plot for the pea samples obtained from PCA accounted for $86 \%$ of explained variance (Figure 1a). Results from the ANOVA model computed on DA data for the sweetcorn samples showed a significant sample effect for 15 of the 19 attributes. The first two components of the score plot for sweetcorn obtained from PCA accounted for $82 \%$ of explained variance (Figure 2a).

\section{$\underline{2.2 \text { Samples evaluation by consumers }}$}

\subsubsection{Participants}

Elderly people were recruited at elderly care institutions and leisure facilities for the elderly in Florence (Italy, IT) and Lille (France, FR). Subjects were recruited to cover the different age groups of the elderly population (Forman et al., 1992), with a group aged from 65 to 69 years (Young old), a group aged from 70 to 79 years (Middle old) and a group aged over 80 years (Very old). Demographic details of the participants as a function of country and age segment are reported in Table 1 . All elderly participants had no medical conditions and were able to independently perform the test. Participants aged from 18 to 64 years (Adults) were also recruited in the Florence area as control groups, respectively for the evaluation of the pea samples ( 34 females, 21 males, mean age 28.0 years) and sweetcorn samples ( 38 females, 21 males, mean age 36.3 years). Appropriate health and safety considerations, together with a risk assessment protocol, were carried out prior to the commencement of the research. Individual written informed consent was obtained from participants.

\subsubsection{Experimental procedure}

Pea and sweetcorn samples were evaluated in two independent sessions. The experiment took place in public spaces such as canteens or common rooms. Tests were conducted individually and social 
interaction was not allowed. The experimental procedure consisted of three steps: 1. Liking test, 2. Collection of Questionnaire data, 3. Sorting task.

Liking test: Participants were provided with individual trays with 11 or 9 three-digit coded pea or sweetcorn samples (10 pea samples plus a replicate; eight sweetcorn samples plus a replicate). Twentyfive grams of product were used for each sample. Peas were presented at $54-56{ }^{\circ} \mathrm{C}$ in a foam cup sealed with a plastic top. Sweetcorn samples were presented in a plastic cup at room temperature. Presentation order was randomized across participants. Participants were asked to look at the appearance, and to smell and taste a teaspoon of each sample, then they were asked to rate their liking on a 9-point category scale (1: dislike extremely- 9: extremely like). Participants were asked to rinse their mouth with water before starting the evaluation and after each sample.

Questionnaire: After completing the liking task, participants filled in a questionnaire consisting of two sections: 1. Demographic characteristics (age, gender); 2. Familiarity with pea and sweetcorn products on a 5 point category scale (1: "I do not recognize the product", 2: "I recognize the product, but I have not tasted it", 3: "I have tasted, but I do not use the product", 4: "I occasionally eat the product" and 5: “I regularly eat the product) (Bäckström et al., 2004). In this scale, scores increase from lexical/visual knowledge (scores 1 and 2), to a taste experience not associated with consumption (score 3 ) and to frequency of consumption (scores 4 and 5 ). Sorting task: In the last part of the session, subjects were provided with a new tray with 11 or 9 threedigit coded pea or sweetcorn samples (ten pea samples plus a replicate; eight sweetcorn samples plus a replicate). Subjects were asked to observe, smell and taste the samples and then to group them according to their similarities, using their own criteria. Subjects were allowed to taste each sample more than once and were asked to note their groupings, and the characteristics of each group, individually. Subjects were asked to rinse their mouth with water before starting evaluation and after each sample.

\subsection{Data analysis}

\subsubsection{Liking data}

Liking data obtained from each product were submitted to a PCA in order to obtain a preference map for each country and each age segment of participants. The reliability of the obtained maps was assessed considering the closeness of the blind duplicate samples (Lawless \& Heymann, 2010), measured considering the reciprocal of the percentage ratio of distance ( $\mathrm{Dr} \%$ ), computed as the ratio between the distance of the two replicated samples and the distance of the two most distant samples on the map (Torri et al., 2013).

\subsubsection{Questionnaire}

Individual data on vegetable familiarity were transformed: responses 1, 2 and 3 were included in the category 'Un-familiar' (UFs), while responses 4 and 5 were included in the category 'Familiar' (Fs). Significant differences in number of Fs and UFs between countries and vegetable typology were assessed using Fisher's exact test within each age segment and in total.

For each subject a distance matrix was generated, where a value of 0 between a row and a column indicates that the assessor put the samples together, whereas a value of 1 indicates that samples were not put together. Individual distance matrices were submitted to DISTATIS (Abdi et al., 2007), a generalization of classical multidimensional scaling that considers individual sorting data. DISTATIS was computed for each country and each age segment, in order to obtain a spatial representation of product 
similarity in which products are represented by points on a map. The points are arranged in this representation so that the distances between pairs of points reflect the similarities among the pairs of stimuli. The adoption of DISTATIS also allowed consideration of the individual variability in the process of categorization, in this way providing a spatial representation less influenced by assessors that behave differently from others. The reliability of the obtained maps was assessed considering the reciprocal of the Dr\%. The hierarchical cluster analysis with Ward's criterion was performed on samples coordinated on the first two components to identify groups of samples in each configuration (Lelièvre et al., 2009).

\subsubsection{Maps comparison}

The similarity of the first two dimensions of the maps was assessed considering the RV coefficient (Robert \& Escoufier, 1976). The RV coefficient is a measure of the similarity between two factorial configurations, which takes the value of 0 if the configurations are uncorrelated, and the value of 1 if the configurations are homothetic. The minimum RV value that has been considered as an indicator of good agreement between sample configurations ranges from 0.65 to 0.85 (Vidal et al., 2014), therefore a cut-off of 0.75 was considered for this study. With respect to each vegetable, the RV coefficient and its statistical significance was computed for all combinations between the compromise maps from DISTATIS on FST data (categorization maps), the score plots from PCA on DA data (sensory maps) and the score plots from PCA on liking data (preference maps), within each country and age segment. Considering that RV coefficients put particular emphasis on the component with the largest variance, the similarity between maps was assessed also considering a visual evaluation of the configurations as suggested in Tomic et al. (2015).

All analyses on consumer data were conducted with the R Statistics Package version 3.2.1 (R Core Team, 2015) using the FactoMineR package (Le et al., 2008) and the DistatisR package (Beaton et al., 2013).

\section{Results}

\subsection{Familiarity for pea and sweetcorn products across countries and age groups}

In order to evaluate the familiarity for pea and sweetcorn products, differences in the distribution of Fs and UFs subjects between vegetable typology were investigated in each country and age group independently (Table 2). The pea typology was in general highly familiar, while the sweetcorn was less familiar irrespective to country and age group. The only exception is in the Very old French subjects, where the lower number of subjects involved in the evaluation of pea products doesn't allow observation of the tendency that emerged in the other age groups. Also in the Adult control group, familiarity with

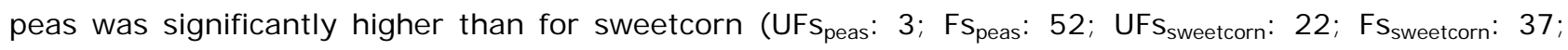
$\mathrm{p}<0.001)$. In order to evaluate if the two countries share the same familiarity with peas and sweetcorn, differences in the distribution of Fs and UFs subjects between countries were investigated for each vegetable typology and age group independently (Table 3). No significant differences between Italy and France were found for peas, but a lower number of Fs were found in Italy compared to France for sweetcorn. Considering the distributions in the different age groups, number of Fs in Italy for sweetcorn were significantly lower than in France only in the case of Very old subjects. Considering the subjects inside each age group and irrespective to the country of origin, for peas the percentage of Fs was constant from Adults to Very old subjects (Adults: 94.5\%; Young old: 98.3\%; Middle old: 95.7\%; Very old: $92.8 \%)$. In the case of sweetcorn, a similar trend was found, excepting the Very old subjects 


\subsection{Similarity among categorization, preference and sensory maps}

\section{$260 \quad 3.2 .1$ Comparison across countries}

The categorization maps obtained from the two countries are shown in Figure 3. In the case of peas, the maps from Italian and French respondents were very similar in terms of relative categorization of the samples. Furthermore, in both maps the replicated samples fall in the same group as expected. Spatial configurations of sweetcorn samples were different in the two countries and the sample groups were formed from different samples. Nevertheless, in both configurations the replicate samples fall in the same group thus still indicating the reliability of the configurations. The similarity between categorization maps from Italian and French respondents expressed as RV coefficients is reported in Table 4 independently for each product. For peas, the correlation of FST configurations between countries is high ( $R V=0.95$, $\mathrm{p}<0.001)$. Conversely, for sweetcorn the correlation of FST configurations between countries is low $(R V=0.54, p<0.05)$, highlighting the different criteria used to perform the categorization of samples in the two countries.

The comparison of preference maps from pea samples between countries (Figure 4) resulted in a RV coefficient of $0.89(p<0.001)$, showing a general agreement on the value of hedonic properties when discriminating between samples. In the case of sweetcorn the comparison between preference maps resulted in a low level of similarity $(R V=0.61, p<0.01)$, suggesting that different sensory properties drive the liking for sweetcorn among I talian and French population. In order to evaluate the weight of sensory and hedonic dimensions on the process of categorization, the categorization map of each country was compared with the relevant sensory and preference maps (Table 4). For the pea samples, the categorization maps from both countries were highly correlated with the sensory maps and also with the corresponding preference map. For sweetcorn, the spatial configuration from FST was poorly correlated with the sensory map, reaching a maximum of the critical RV value of $0.75(p<0.01)$ in the French group. This suggests that subjects gave a different weight to the sensory attributes that determinate the dimensions of the categorization map, particularly in the case of the Italians $(\mathrm{RV}=0.57, \mathrm{p}<0.05)$. Also the correlation between categorization maps and preference maps revealed a poor correlation between the two configurations in both countries.

\subsubsection{Comparison across age segments}

In order to study the effect of ageing on the drivers of categorization and sorting performance, sorting data and liking data for both countries were merged by age group and data analysis was carried out independently for each age segment. A characterization of each age segment is reported in Table 1. Categorization and preference maps from the control group of Adults were used as reference. The categorization maps and the preference maps obtained from the four age groups are shown in Figures 5 and 6 , respectively. For the pea samples, the FST groups were formed by the same samples in each age group, with the exception of sample B in the Very old segment. Sweetcorn groups were formed by different samples in each age segment. Replicated samples always fell in the same group both for pea and sweetcorn samples irrespective to age, confirming the reliability of the configurations. The level of similarity between categorization, preference and sensory maps as a function of aging is reported in Figure $7 \mathrm{a}$ for peas and in Figure $7 \mathrm{~b}$ for sweetcorn. The following comparisons were considered: 1. The categorization map from the reference group of Adults versus each categorization map from the three elderly age groups; 2 . Categorization maps from Adults and the three elderly age 
groups versus the sensory map; 3. Categorization maps from Adults and from the three elderly age groups versus the relative preference maps.

303 Considering the pea samples, the correlation between the categorization maps from the Adults and each elderly group is high in the Young old $(R V=0.97, p<0.001)$ and Middle old segment $(R V=0.97, p<0.001)$, suggesting a strong similarity in the categorization of pea samples. A slight decrease in similarity can be found in the Very old segment $(R V=0.82, p<0.001)$, but the categorization of samples remains comparable. In the case of the sweetcorn samples, the maps follow a completely different pattern. The correlation between the categorization maps from the Adults and each elderly group decreases to Young old $(R V=0.68, p<0.01)$, Middle old $(R V=0.53, p<0.05)$ and Very old $(R V=0.29, p>0.05)$ segments. This evidence suggests that for this typology of product, the criteria used in categorizing the samples varies during the ageing process, with an overall effect on sorting configuration. Taking into consideration the similarity between the categorization maps and the sensory map, in the case of peas it is possible to see that the sensory dimension is highly important in each age segment (minimum RV value: Very Old segment $(\mathrm{RV}=0.81, \mathrm{p}<0.001)$ ). Conversely, in the case of sweetcorn the similarity between the categorization maps and the sensory map decreases from Adults to the Very old, the latter with the minimum level in similarity $(R V=0.39, p>0.05)$.

Concerning the similarities between the categorization maps and the preference maps, in the case of peas, the results show little differences in the value of the hedonic dimension in the presented samples from Adults to the Very old segment. Moreover, the contribution of the hedonic dimension to the categorization process remains lower than the sensory dimension in each age segment, with a maximum $R V$ value reached in the Middle old segment $(R V=0.77, p<0.001)$. A similar tendency was found for sweetcorn, with a minimum similarity reached in the Very old segment $(R V=0.47, p>0.05)$.

\subsubsection{Maps reliability within each age segment}

The performance of FST during ageing was further explored by considering the reliability of the maps generated from each age segment, using the ratio of distances between the two replicated samples. The Dr\% of categorization maps and preference maps are reported in Figures $8 a$ and $8 b$, respectively for each age class and vegetable category. In this plot, the closer the two replicated samples are on the map the higher the Dr\% value and thus the map reliability. For the pea samples, both the categorization and preference maps showed a high level of reliability in each age segment. For the sweetcorn a high level of reliability was found in each age group only for the categorization maps, while for the preference maps the reliability decreases with age. In particular for the pea samples the lowest Dr\% of the categorization maps was reached in the Middle old subjects ( $\mathrm{Dr} \%=86.0 \%$ ), while in the preference maps, the minimum Dr\% was reached in Very old subjects $(\mathrm{Dr} \%=80.6 \%)$. Considering the sweetcorn samples, the FST produced highly reliable maps in each age segment, with a minimum Dr\% reached in the Adult group (Dr\% $=79.5 \%)$. A different performance was obtained for the liking task, where the reliability of the preference maps decreased from the Adults to the Very old subjects, with a minimum Dr\% in the Very old group $(D R \%=49.3 \%)$.

\section{Discussion}

In order to study the role of sensory and hedonic dimensions in the process of categorization, samples of pea and sweetcorn were selected in order to cover as much sensory space as possible of both vegetable 
typologies. The DA validated the sensory variability of the experimental sample sets, where the selected samples of pea and sweetcorn varied significantly on the quality and intensity of several descriptors relevant to different sensory modalities.

Moreover pea and sweetcorn samples were chosen in order to study the effect of familiarity on the process of categorization. Peas were chosen due to their long presence in European food culture, while sweetcorn was characterized by a recently introduction to the continent. Our results confirm a high familiarity with peas in each country and age group considered in the study. Conversely, in the case of sweetcorn, each country and age group showed poor familiarity, most notable in the Italian older adults. Thus the results confirm the higher familiarity of pea compared to sweetcorn and a comparable familiarity toward the vegetable typologies between the two countries, with the only exception being the older segment involved in the sweetcorn evaluation. Moreover, considering the different age groups irrespective of country, familiarity towards peas was constant with age, and a similar trend was found for sweetcorn, excepting in the Very old subjects.

\subsection{The performance of the free sorting task among countries and age groups}

The differences in familiarity toward the tested vegetables affected the FST categorization maps in both countries. In the case of the familiar product, the configuration and grouping of samples from FST was comparable between the countries. Conversely, in the case of the unfamiliar product, the similarity between the categorization maps was clearly lower than in the previous case, indicating the use of different criteria in the categorization of samples. In order to study how the process of categorization may change during ageing we merged French and Italian subjects considering that the familiarity toward pea and sweetcorn products was generally comparable between countries inside each age group.

Considering the familiar vegetable, ageing weakly affected the categorization criteria as indicated by the high level of similarity between the categorization maps among the different age groups. Moreover the categorization maps showed a high level of reliability in all age groups, suggesting that categorization performance remains high during ageing. Furthermore, the high level of similarity between the categorization maps from the Adult reference group and each elderly group suggests that is possible to infer the categorization criteria of a healthy elderly population even using adult subjects when a comparable level of familiarity is shared. In the case of the unfamiliar vegetable the map obtained from FST significantly changed across age groups, thus indicating that the criteria used in the classification of samples varied during ageing, possibly because of the lower familiarity with the product. Despite the different spatial configurations, the reliability of the maps was high and comparable in each age group, confirming good performances in the categorization task. Therefore also using an unfamiliar vegetable, the FST remains a suitable method for use among healthy older adults. However, the low level of similarity between the categorization map from Adults and the categorization maps from each elderly group indicates that reliable information on categorization criteria can be inferred only by considering the age group of interest. Overall the results suggest that FST allowed the detection of differences in sample categorization in the different age groups of the elderly population and the different countries, and so is applicable for older adults. The present research therefore corroborates the good applicability of sorting methodology with healthy older adults as reported by Withers et al. (2014).

\subsection{The role of sensory and hedonic dimensions in the categorization of vegetables}


The study showed that the sensory dimension is the main driver of categorization in the case of the familiar product. In fact the categorization maps depict the same similarities and differences among vegetable samples described by the trained panel with DA, irrespective of the country and the age group. The ability of the FST to generate maps comparable with the sensory maps from DA was already reported in adult subjects (Faye et al., 2004; Saint-Eve et al., 2004) and in the present study this was confirmed also in the elderly population in the case of a high familiar product. Considering the unfamiliar vegetable, the comparison between the categorization maps and the sensory maps highlighted a gradual decrease in similarity with age, thus indicating a reduction in the influence of the sensory dimension in the process of categorization. However this tendency may also mean that the categorization of sweetcorn samples does not reflect differences and similarities in sensory descriptors as perceived by the trained assessors in DA, an aspect that in an elderly respondent may be due to an impaired perception (Schubert et al., 2012) or may be due to the salience of different sensory attributes, such as mouthfeel characteristics (Forde \& Delahunty, 2004). The other potential driver of categorization investigated in the study was the hedonic dimension. The categorization of the familiar product was more influenced by the sensory dimension than the hedonic one, an aspect already reported in research on foods categorization with adults (Ballester et al., 2008; Chollet \& Valentin, 2000). However the hedonic pattern of the samples still partially superimposes the configurations resulting from the FST in each age group, suggesting that is possible to obtain an indication of the general liking using categorization maps. In the case of the unfamiliar product, a reduction in similarity between the categorization map and the preference map was detected from Adults to Young old to Very old subjects. In this case, the tendency seems to be due to an issue related to the performance of the methodology as the reliability index of the preference maps decreases with age, reaching a low level in particular among Very old subjects.

\subsection{Sensory-cognitive interaction in flavour building}

It is noteworthy to consider how in the case of the familiar product the drivers of sample categorization are shared among Adults and the older age groups, while in the case of the unfamiliar product they change during ageing. The differences in the categorization of the familiar and unfamiliar vegetable may be due to the use of different processes in products representation. In fact the categorization can be the results of two distinct cognitive paths, namely similarity-based processes (Juslin et al., 2003) and rulebased processes (Ashby et al., 1998). Similarity-based processes rely on exemplar retrieval from memory, where objects are categorized on the basis of their similarity to already known exemplars. On the other hand, rule-based processes are based on the integration of cues (i.e., the characteristics of the objects). Research reports that in categorization tasks, adult subjects tend to rely on similarity-based processes (von Helversen et al., 2010) due to the lower cognitive demand in respect to the rule-based processes. It is possible to hypothesize that consumers may use similarity-based processes when a familiar product is evaluated, with the effect of building the perception of a product on the base of perceptive elements that subjects learned to associate with specific sensory exemplars. An empirical example of this process is provided by Morot et al. (2001), where the red coloration of a white wine led the assessor to elicit smell attributes characteristic of red wines, therefore demonstrating the use of topdown cognitive processes in the building of wine flavour. On the other hand, in the evaluation of an unfamiliar product the absence of previous knowledge may push subjects to use rule-based processes, 
assumptions therefore suggest that among older adults the lack of previous experience with the unfamiliar product led to the building of perceptions mainly using surface sensory properties, that may change during the ageing due to possible sensory impairments. In the case of the familiar product the perceptive information was combined with cognitive information from previous experience, thus compensating the eventual perceptive losses that may occur in this population segment.

\section{Conclusions}

In the context of better understanding the perception of healthy foods among different age segments of older adults, this research aimed to explore the performance of free sorting task methodology and the drivers of categorization among healthy older adults of two European countries, France and Italy. The results confirm that the free sorting task is a suitable and reliable method to use with healthy older adults, that is able to detect differences in the categorization of stimuli even among the more aged representatives of this segment of the population. Age influences familiarity toward the tested product, and familiarity was the main factor that affected categorization maps and the information that can be extracted from them. Categorization maps from a familiar product can be potentially used to obtain reliable information of sensory and hedonic dimensions, while maps obtained from an unfamiliar product depict mainly the sensory variability. This suggests that when older adults are encouraged to elicit sensory and hedonic terms to describe the formed groups of a familiar product it may be possible to obtain an indication of the sensory properties of the samples and the general direction of liking. Moreover the study highlighted that among healthy older adults, familiarity toward a food may play a role in flavour building, where in the case of a familiar product the cognitive information from previous experiences of consumption seems to compensate for the sensory loss that older adults may experience.

\section{Acknowledgements}

The research leading to these results received funding from the People Programme (Marie Curie Actions) of the European Union's Seventh Framework Programme for research, technological development and demonstration under grant agreement $n^{\circ}$ [612326] "VeggiEAT - To critically evaluate acceptability through individual and environmental characteristics across the lifespan in institutional food service".

\section{References}

Abdi, H., Valentin, D., Chollet, S., \& Chrea, C. (2007). Analyzing assessors and products in sorting task:

Appleton, K. M., Hemingway, A., Saulais, L., Dinnella, C., Monteleone, E., Depezay, L., Morizet, D.,

Perez-Cueto, F. J. A., Bevan, A., \& Hartwell, H.(2016). Increasing vegetable intakes: rationale and systematic review of published interventions. European Journal of Nutrition, 55(3), 869-896.

Appleton, K. M. (2016). Barriers to and facilitators of the consumption of animal-based protein-rich foods in older adults. Nutrients, 8, 187. 
of multiple systems in category learning. Psychological Review, 105, 442-481.

Bäckström, A., Pirttilä-Backman, A.M., \&Tuorila, H. (2004). Willingness to try new foods as predicted by social representations and attitude and trait scales. Appetite,43(1), 75-83.

Ballester, J., Patris, B., Symoneaux, R., \& Valentin, D. (2008). Conceptual vs perceptual wine spaces: does expertise matter? Food Quality and Preference, 19, 267-276.

Barylko-Pikielna, N., Matuszewska, I., Jeruszka, M., Kozlowska, K., Brzozowska, A., \& Roszkowski, W. (2004). Discriminability and appropriateness of category scaling versus ranking methods to study sensory preferences in elderly. Food Quality and Preference, 15(2), 167-175.

Beaton, D., Fatt, C. C., \&Abdi, H. (2013). DistatisR: DiSTATIS Three Way Metric Multidimensional Scaling. R package version 1.0. http://CRAN.R-project.org/package=DistatisR

Blancher, G., Chollet, S., Kesteloot, R., Nguyen Hoang, D., Cuvelier, G., \& Sieffermann, J. M. (2007). French and Vietnamese: how do they describe texture characteristics of the same food? A case study with jellies. Food Quality and Preference, 18(3), 560-575.

Bucher, T., Collins, C., Diem, S.,\& Siegrist, M. (2016). Adolescents' perception of the healthiness of snack. Food Quality and Preference, 50, 94-101.

Cartier, R., Rytz, A., Lecomte, A., Poblete, F., Krystlik, J., Belin, E., \& Martin, N. (2006). Sorting procedure as an alternative to quantitative descriptive analysis to obtain a product sensory map. Food Quality and Preference, 17, 562-571.

Chollet, S., \& Valentin, D. (2000). Le degreé d'expertise a-t-il une influence sur la perception olfactive? Quelques éléments de réponse dans le domaine du vin. L'Année Psychologique, 100, 11- 36.

Chrea, C., Valentin, D., Sulmont-Rossé, C., Ly Mai, H., Hoang Nguyen, D., \& Abdi, H. (2004). Culture and odor categorization: agreement between cultures depends upon the odors. Food Quality and Preference, $15,669-679$.

Cruz-Jentoft, A. J., Baeyens, J. P., Bauer, J. M., Boirie, Y., Cederholm, T., Landi, F., et al. (2010). Sarcopenia: European consensus on definition and diagnosis: Report of the European Working Group on Sarcopenia in Older People. Age and Ageing, 39(4), 412-423.

Deegan, C., Koivisto, L., Näkkilä, J., Hyvönen, L., \& Tuorila, H. (2010). Application of a sorting procedure to greenhouse-grown cucumbers and tomatoes. LWD - Food Science and Technology, 43, 393-400.

Dermiki, M., Mounayar, R., Suwankanit, C., Scott, J., Kennedy, O. B., Mottram, D. S., Gosney, M. A., Blumenthal, H., \& Methven, L. (2013). Maximizing umami taste in meat using natural ingredients: effects on chemistry, sensory perception and hedonic liking in young and old consumers. Journal of the Science of Food and Agriculture, 93(13), 3312-3321. 
Dermiki, M., Willway, J., Sargent, L., Kidman, J., Anderson, C., Kennedy, O. B., \& Gosney, M. A., \&

Methven, L. (2014). Preference and consumption of a taste enhanced meat meal by older hospital patients: a pilot study. Nutrition and Aging, 2(1), 69-75.

Dinnella, C., Morizet, D., Masi, C., Cliceri, D., Depezay, L., Appleton, K. M., Giboreau, A., Perez-Cueto, F. J. A., Hartwell, H., \& Monteleone, E. (2016). Sensory determinants of stated liking for vegetable names and actual liking for canned vegetables: a cross-country study among European adolescents. Appetite (2016), doi: 10.1016/j.appet.2016.08.110

Faye, P., Brémaud, D., Durand Daubin, M., Courcoux, P., Giboreau, A.,\& Nicod, H. (2004). Perceptive free sorting and verbalization tasks with naïve subjects: an alternative to descriptive mappings. Food Quality and Preference, 15, 781-791.

Forde, C. G., \& Delahunty, C. M. (2004). Understanding the role cross-modal sensory interactions play in food acceptability in younger and older consumers. Food Quality and Preference, 15, 715-727.

Forman, D. E., Berman, A. D., McCabe, C. H., Baim, D. S., \& Wei, J. Y. (1992). PTCA in the elderly: the "young-old" versus the "old-old". J ournal of the American Geriatrics Society, 40(1), 19-22.

GBD 2013 DALYs and HALE Collaborators (2015). Global, regional, and national disability-adjusted life years (DALYs) for 306 diseases and injuries and healthy life expectancy (HALE) for 188 countries, 19902013: quantifying the epidemiological transition. Lancet, 386, 2145-2191. S., Lindberg, U., \& Risvik, E. (2016). Health and quality of life in an aging population - food and beyond. Food Quality and Preference, 47, 166-170.

Hickson, M. (2006). Malnutrition and ageing. Postgraduate Medical J ournal, 82, 2-8.

Juslin, P., Olsson, H., \& Olsson, A. C. (2003). Exemplar effects in categorization and multiple-cue judgment. J ournal of Experimental Psychology: General, 132, 133-156.

Kapica, C., \& Weiss, W. (2012). Canned fruits, vegetables, beans and fish provide nutrients at a lower cost compared to fresh, frozen or dried. Conference: Experimental Biology Meeting Location: San Diego, CA APR 21-25, 2012; FASEB J ournal, 26. Science \& Business Media. 
Le, S., Josse, J., \& Husson, F. (2008). FactoMineR: An R Package for Multivariate Analysis.Journal of Statistical Software, 25(1), 1-18.

Lee, S. M., Kim, S., Guinard, J., \& Kim, K. (2016). Exploration of flavor familiarity effect in Korean and US consumers' hot sauces perceptions. Food Science and Biotechnology, 25(3), 745-746.

565

566

567

568

569

570

571

572

573

Lelièvre, M., Chollet, S., Abdi, H.,\& Valentin, D. (2009). Beer-trained and untrained assessors rely more on vision than on taste when they categorize beers. Chemosensory Perception, 2, 143-153.

Methven, L., Jiménez-Pranteda, M. L., \& Lawlor, J. B. (2016). Sensory and consumer science methods used with older adults: a review of current methods and recommendations for the future. Food Quality and Preference, 48, 333-344.

Mingioni, M., Mehinagic, E., Laguna, L., Sarkar, A., Pirttijärvi, T., Van Wymelbeke, V., Artigas, G., Chen, J., Kautola, H., Järvenpää, E., Mäenpää, T., Tahvonen, R., Grabska-Kobylecka, I., \&Maitre, I. (2016). Fruit and vegetables liking among European elderly according to food preferences, attitudes towards food and dependency. Food Quality and Preference, 50, 27-37.

Morizet, D., Depezay, L., Combris, P., Picard, D., \& Giboreau, A. (2012). Effect of labeling on new vegetable dish acceptance in preadolescent children. Appetite, 59, 399-402.

Morot, G., Brochet, F., \& Dubourdieu, D. (2001). The color of odors. Brain and Language, 79, 309-320.

Næs, T., Brockhoff, P. B., \& Tomic, O. (2010). Statistics for sensory and consumer science. Chichester. John Wiley \& Sons. Ltd.

Nestrud, M. A., \& Lawless, H. T. (2010). Perceptual mapping of apples and cheeses using projective mapping and sorting. J ournal of Sensory Studies, 25(3), 390-405.

Nyberg, M., Olsson, V., Pajalic, Z., Örtman, G., Håkan, S. A., Blücher, A., Wendin, K., \& Westergren, A. (2015). Eating difficulties, nutrition, meal preferences and experiences among elderly. A literature overview from a Scandinavian context. J ournal of Food Research, 4(1), 22-37.

Pelt, J.M. (1993). In: Des legumes. Editions FAYARD

R Core Team (2015). R: A language and environment for statistical computing. R Foundation for Statistical Computing, Vienna, Austria. URL http://www.R-project.org/

Robert, P., \& Escoufier, Y. (1976). A unifying tool for linear multivariate statistical methods: The RVcoefficient. Journal of the Royal Statistical Society. Series C (Applied Statistics), 25, 257-265.

Robine, J., \& Camboise, E. (2013). Healthy life expectancy in Europe. Population \& Societies - Monthly bulletin of the French National Institute for Demographic Studies, 499. 
Rosch, E., \& Lloyd, B. B. (1978). Cognition and categorization. Hillsdale, NJ: Lawrence Erlbaum.

Roubenoff, R. (2000). Sarcopenia and its implications for the elderly. European Journal of Clinical

Schubert, C. R., Cruickshanks, K. J., Fischer, M. E., Huang, G. H., Klein, B. E., Klein, R., Pankow, J. S., \& Nondahl, D. M. (2012). Olfactory impairment in an adult population: the beaver dam offspring study. Chemical Senses, 37, 325-334.

Saint-Eve, A., Paçi Kora, E., \& Martin, N. (2004). Impact of the olfactory quality and chemical complexity of the flavouring agent on the texture of low fat stirred yogurts assessed by three different sensory methodologies. Food Quality and Preference, 15, 655-668.

Solomon, G. E. A. (1997). Conceptual change and wine expertise. The Journal of the Learning Sciences, 6(1), 41-60.

Santosa, M., Abdi, H., \& Guinard, J. (2010). A modified sorting task to investigate consumer perceptions of extra virgin olive oils. Food Quality and Preference, 21(7), 881-892.

Song, X., Giacalone, D., Bølling Johansen, S. M., Frøst, M. B., \& Bredie, W. L. P. (2016). Changes in orosensory perception related to aging and strategies for counteracting its influence on food preferences among older adults. Trends in Food Science \& Technology, 53, 49-59.

Tomic, O., Berget, I.,\& Næs, T. (2015). A comparison of generalised procrustes analysis and multiple factor analysis for projective mapping data. Food Quality and Preference, 43, 34-46.

Torri, L., Dinnella, C., Recchia, A., Næs, T., Tuorila, H., \& Monteleone, E. (2013). Projective mapping for interpreting wine aroma differences as perceived by naïve and experienced assessors. Food Quality and Preference, 29, 6-15.

United Nations, Department of Economic and Social Affairs, Population Division (2015). World Population Ageing 2015 (ST/ESA/SER.A/390).

Varela, P., \& Salvador, A. (2014). Structured sorting using pictures as a way to study nutritional and hedonic perception in children. Food Quality and Preference, 37, 27-34. Assessment of global and individual reproducibility of projective mapping with consumers. Journal of Sensory Studies, 29, 74-87. 
648 Withers, C., Methven, L., Qannari, E. M., Allen, V. J., Gosney, M. A., \& Macfie, H. J. H. (2014). Taxonomic 649 free sorting: a successful method with older consumers and a novel approach to preference mapping. 650 Journal of Sensory Studies, 29, 182-189.

651

652

653

654

655

656

657

658

659

660

661

662

663

664

665

666

667

668

669

670

671

672

673

674

675

676

677

678

679

680

681

682

683

684

685

686

687

688 
Figure 1 (a-b). Sensory maps: Score plot (a) and correlation loading plot (b) from PCA on panel averages of each significant attribute $(p<0.05)$ describing the sensory properties of pea samples. In the correlation loading plot outer and inner circles on the map represent $100 \%$ and $50 \%$ explained variance respectively.

Figure 2 (a-b). Sensory maps: Score plot (a) and correlation loading plot (b) from PCA on panel averages of each significant attribute $(p<0.05)$ describing the sensory properties of sweetcorn samples. In the correlation loading plot outer and inner circles on the map represent $100 \%$ and $50 \%$ explained variance respectively.

Figure 3. Categorization maps: Compromise map from DISTATIS for pea (left) and sweet corn (right) samples obtained from the free sorting task with French and Italian older adults. The ellipsoids correspond to the clusters identified with hierarchical cluster analysis.

Figure 4. Preference maps: Score plot from PCA for pea (left) and sweet corn (right) samples obtained from the liking task with French and Italian older adults.

Figure 5. Categorization maps: Compromise map from DISTATIS for pea (left) and sweet corn (right) samples obtained from the free sorting task with Adults, Young old, Middle old and Very old segments. The ellipsoids correspond to the clusters identified with hierarchical cluster analysis.

Figure 6. Preference maps: Score plot from PCA for pea (left) and sweet corn (right) samples obtained from the liking task with Adults, Young old, Middle old and Very old segments..

Figure 7 (a-b). RV coefficient values between samples configurations in the first two dimensions of categorization, preference and sensory maps as a function of the age segments and pea (a) and sweetcorn (b) typologies. FST A indicates categorization maps from Adults.

Figure 8 (a-b). Ratio of distances (\%) values for the two replicated samples in the first two dimensions of the categorization and preference maps as a function of the age segments and pea (a) and sweetcorn (b) typologies. 
Table 1. Characteristics of the elderly respondents per product: country, demographics and total number per age group and country. Values in brackets represent standard deviations.

\begin{tabular}{|c|c|c|c|c|c|c|c|c|c|c|}
\hline & \multicolumn{5}{|c|}{ Peas } & \multicolumn{5}{|c|}{ Sweet corn } \\
\hline & \multicolumn{2}{|c|}{ Country } & \multirow[t]{2}{*}{ Total } & \multirow[t]{2}{*}{ Females } & \multirow[t]{2}{*}{ Mean age } & \multicolumn{2}{|c|}{ Country } & \multirow[t]{2}{*}{ Total } & \multirow[t]{2}{*}{ Females } & \multirow[t]{2}{*}{ Mean age } \\
\hline & France & Italy & & & & France & Italy & & & \\
\hline Young old & 78 & 42 & 120 & $65.8 \%$ & $65.7(2.0)$ & 41 & 41 & 82 & $68.3 \%$ & $65.9(1.9)$ \\
\hline Medium old & 18 & 29 & 47 & $65.9 \%$ & $72.8(2.9)$ & 38 & 28 & 66 & $81.8 \%$ & $73.6(3.0)$ \\
\hline Very old & 2 & 25 & 27 & $77.7 \%$ & $85.0(3.5)$ & 19 & 29 & 48 & $87.5 \%$ & $84.1(3.6)$ \\
\hline Total & 98 & 96 & 194 & $67.5 \%$ & $74.5(2.8)$ & 98 & 98 & 198 & $76.7 \%$ & $74.5(2.9)$ \\
\hline Females & $69.3 \%$ & $65.6 \%$ & & & & $79.5 \%$ & $75.5 \%$ & & & \\
\hline Mean age & $67.7(3.2)$ & $72.6(8.9)$ & & & & $72.6(6.8)$ & $74.6(8.4)$ & & & \\
\hline
\end{tabular}


767 Table 2. Distribution of familiar (Fs) and unfamiliar (UFs) subjects testing pea and sweetcorn products as 768 a function of country and age group: occurrences and $p$ values.

\begin{tabular}{|c|c|c|c|c|c|c|}
\hline & \multicolumn{3}{|c|}{ France } & \multicolumn{3}{|c|}{ Italy } \\
\hline & Pea & Sweetcorn & $p$ & Pea & Sweetcorn & $p$ \\
\hline \multicolumn{7}{|l|}{ All subjects } \\
\hline UFs & 1 & 26 & \multirow{2}{*}{$<0.001$} & 4 & 51 & \multirow{2}{*}{$<0.001$} \\
\hline Fs & 97 & 72 & & 92 & 47 & \\
\hline \multicolumn{7}{|l|}{ Young old } \\
\hline UFs & 1 & 8 & \multirow{2}{*}{$<0.001$} & 1 & 16 & \multirow{2}{*}{$<0.001$} \\
\hline Fs & 77 & 33 & & 41 & 25 & \\
\hline \multicolumn{7}{|l|}{ Medium old } \\
\hline UFs & 0 & 11 & \multirow{2}{*}{0.011} & 2 & 14 & \multirow{2}{*}{$<0.001$} \\
\hline Fs & 18 & 27 & & 27 & 14 & \\
\hline \multicolumn{7}{|l|}{ Very old } \\
\hline UFs & 0 & 7 & \multirow{2}{*}{0.533} & 1 & 21 & \multirow{2}{*}{$<0.001$} \\
\hline Fs & 2 & 12 & & 24 & 8 & \\
\hline
\end{tabular}

769

770

771

772

773

774

775

776

777

778

779

780

781

782

783

784

785

786

787

788

789

790

791

792

793

794

795

796

797

798

799 
800 Table 3. Distribution of familiar (Fs) and unfamiliar (UFs) subjects between countries as a function of 801 vegetable product and age group: occurrences and $p$ values.

\begin{tabular}{|c|c|c|c|c|c|c|}
\hline & \multicolumn{3}{|c|}{ Pea } & \multicolumn{3}{|c|}{ Sweetcorn } \\
\hline & France & I taly & $p$ & France & I taly & $p$ \\
\hline \multicolumn{7}{|l|}{ All subjects } \\
\hline UFs & 1 & 4 & \multirow{2}{*}{0.209} & 26 & 51 & \multirow{2}{*}{$<0.001$} \\
\hline Fs & 97 & 92 & & 72 & 47 & \\
\hline \multicolumn{7}{|l|}{ Young old } \\
\hline UFs & 1 & 1 & \multirow{2}{*}{1.000} & 8 & 16 & \multirow{2}{*}{0.088} \\
\hline Fs & 77 & 41 & & 33 & 25 & \\
\hline \multicolumn{7}{|l|}{ Medium old } \\
\hline UFs & 0 & 2 & \multirow{2}{*}{0.517} & 11 & 14 & \multirow{2}{*}{0.123} \\
\hline Fs & 18 & 27 & & 27 & 14 & \\
\hline \multicolumn{7}{|l|}{ Very old } \\
\hline UFs & 0 & 1 & \multirow{2}{*}{1.000} & 7 & 21 & \multirow{2}{*}{0.019} \\
\hline Fs & 2 & 24 & & 12 & 8 & \\
\hline
\end{tabular}

802

803

804

805

806

807

808

809

810

811

812

813

814

815

816

817

818

819

820

821

822

823

824

825

826

827

828

829

830

831

832

833 
834 Table 4. RV coefficient values between samples configurations in the first two dimensions of 835 categorization, preference and sensory maps as a function of the country and vegetable products.

\begin{tabular}{|c|c|c|c|c|c|c|c|c|c|c|}
\hline & \multicolumn{5}{|c|}{ Pea } & \multicolumn{5}{|c|}{ Sweetcorn } \\
\hline & $\begin{array}{l}\text { FST } \\
\text { Italy }\end{array}$ & $\begin{array}{c}\text { FST } \\
\text { France }\end{array}$ & $\begin{array}{l}\text { IPM } \\
\text { Italy }\end{array}$ & $\begin{array}{c}\text { IPM } \\
\text { France }\end{array}$ & DA & $\begin{array}{l}\text { FST } \\
\text { I taly }\end{array}$ & $\begin{array}{c}\text { FST } \\
\text { France }\end{array}$ & $\begin{array}{l}\text { IPM } \\
\text { Italy }\end{array}$ & $\begin{array}{c}\text { IPM } \\
\text { France }\end{array}$ & DA \\
\hline FST I taly & 1 & & & & & 1 & & & & \\
\hline FST France & $0.95^{* * *}$ & 1 & & & & $0.54^{*}$ & 1 & & & \\
\hline IPM I taly & $0.78^{* * *}$ & $0.72^{* * *}$ & 1 & & & $0.52^{*}$ & $0.52^{*}$ & 1 & & \\
\hline IPM France & $0.80^{* * *}$ & $0.75^{* * *}$ & $0.89^{* * *}$ & 1 & & $0.61^{* *}$ & $0.50^{*}$ & $0.61^{* *}$ & 1 & \\
\hline DA & $0.86^{* * *}$ & $0.88^{* * *}$ & $0.80^{* * *}$ & $0.88^{* * *}$ & 1 & $0.57^{*}$ & $0.75^{* *}$ & $0.65^{* *}$ & $0.71^{* *}$ & 1 \\
\hline
\end{tabular}

836

${ }^{*}=p<0.05^{* *}=p<0.01^{* * *}=p<0.001$ 
a.

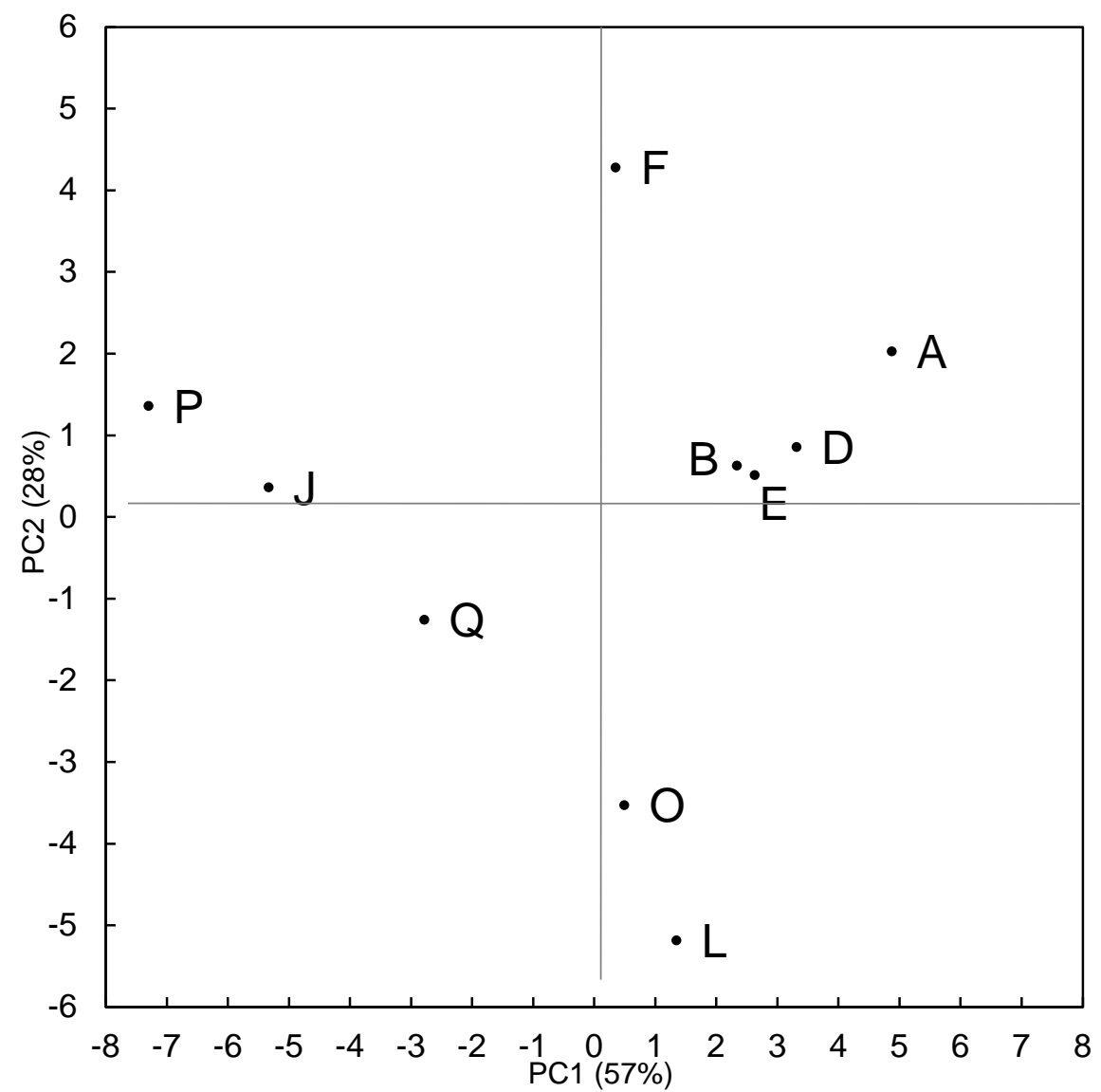

b.

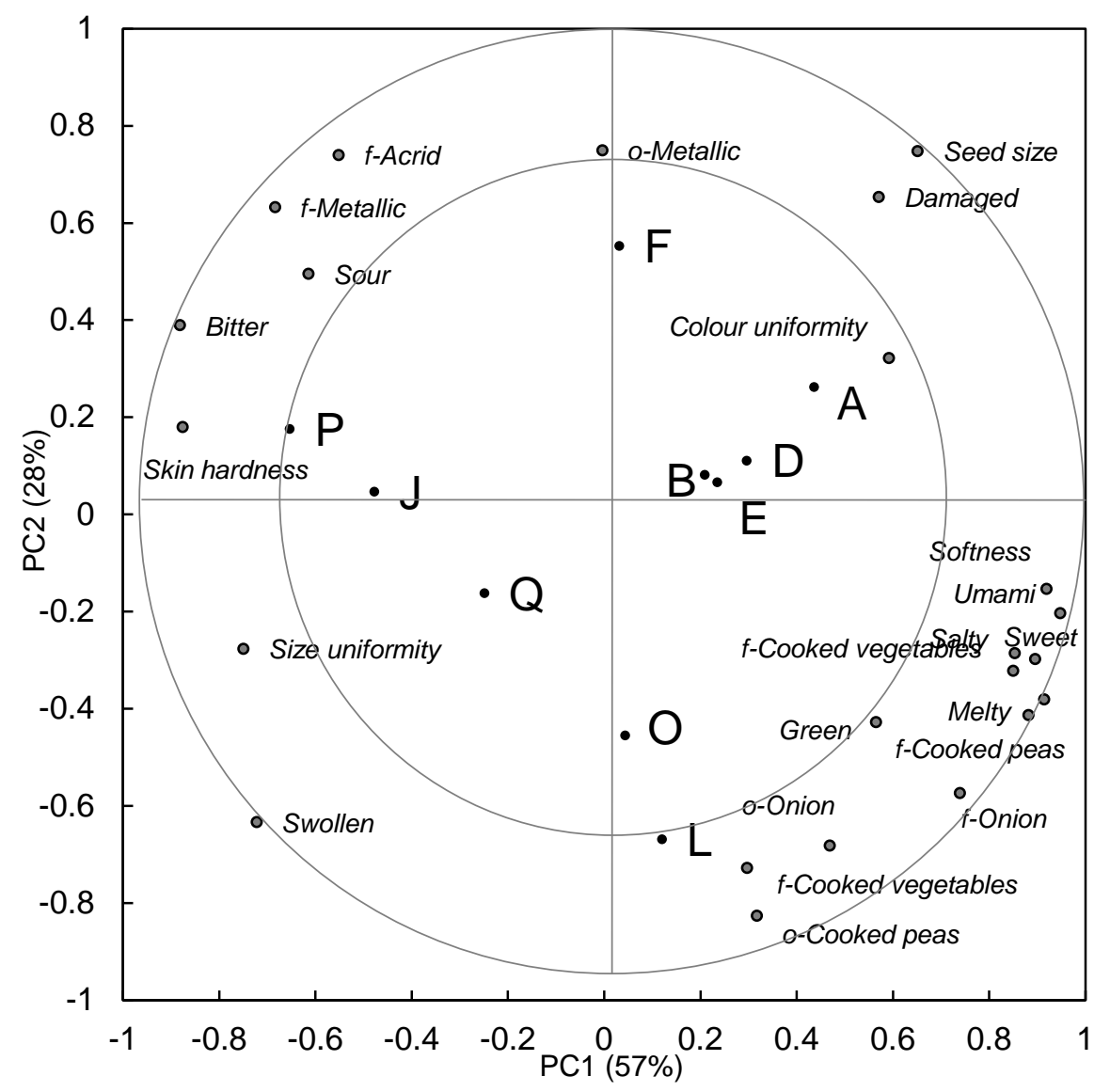


a.

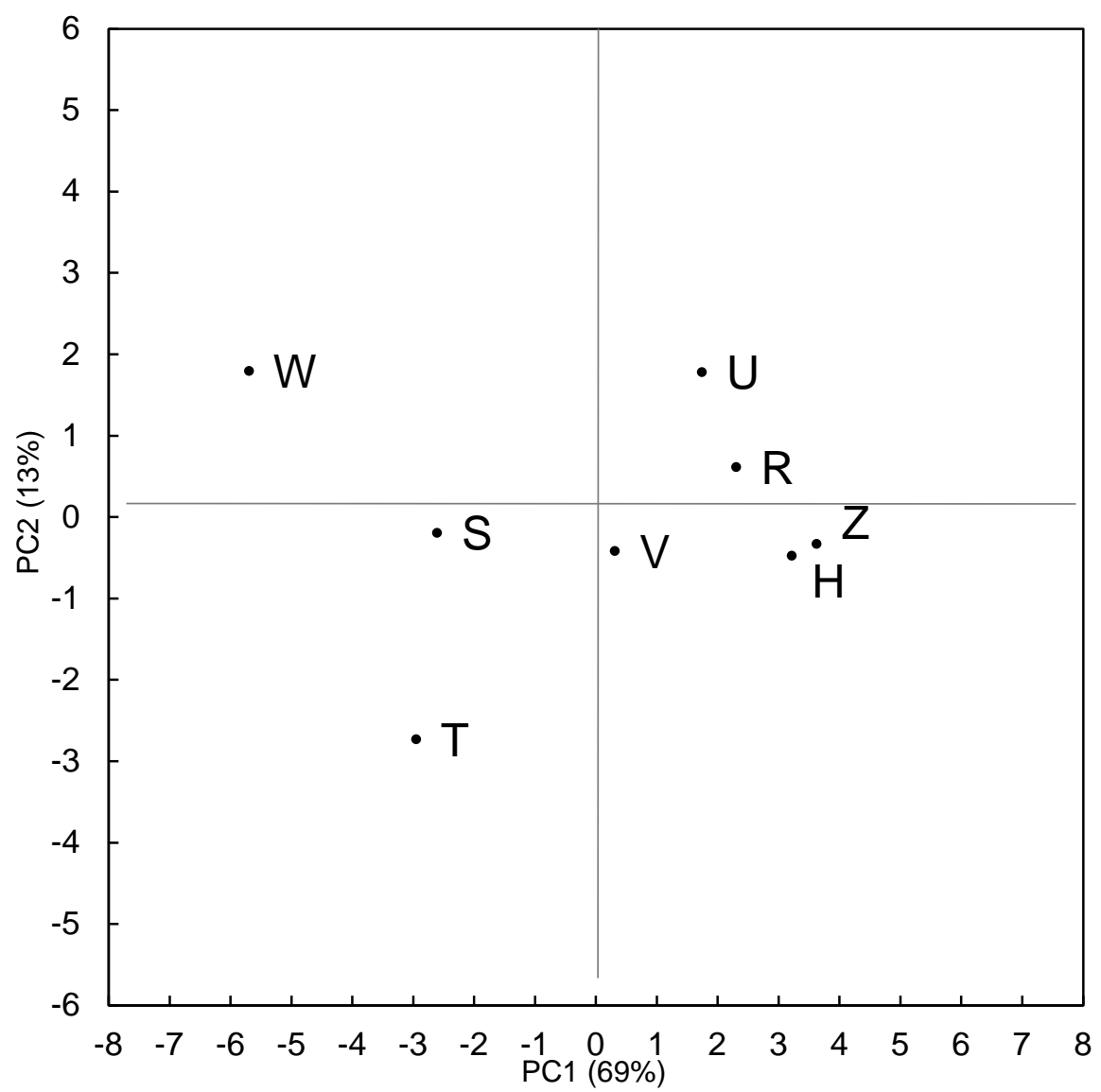

b.

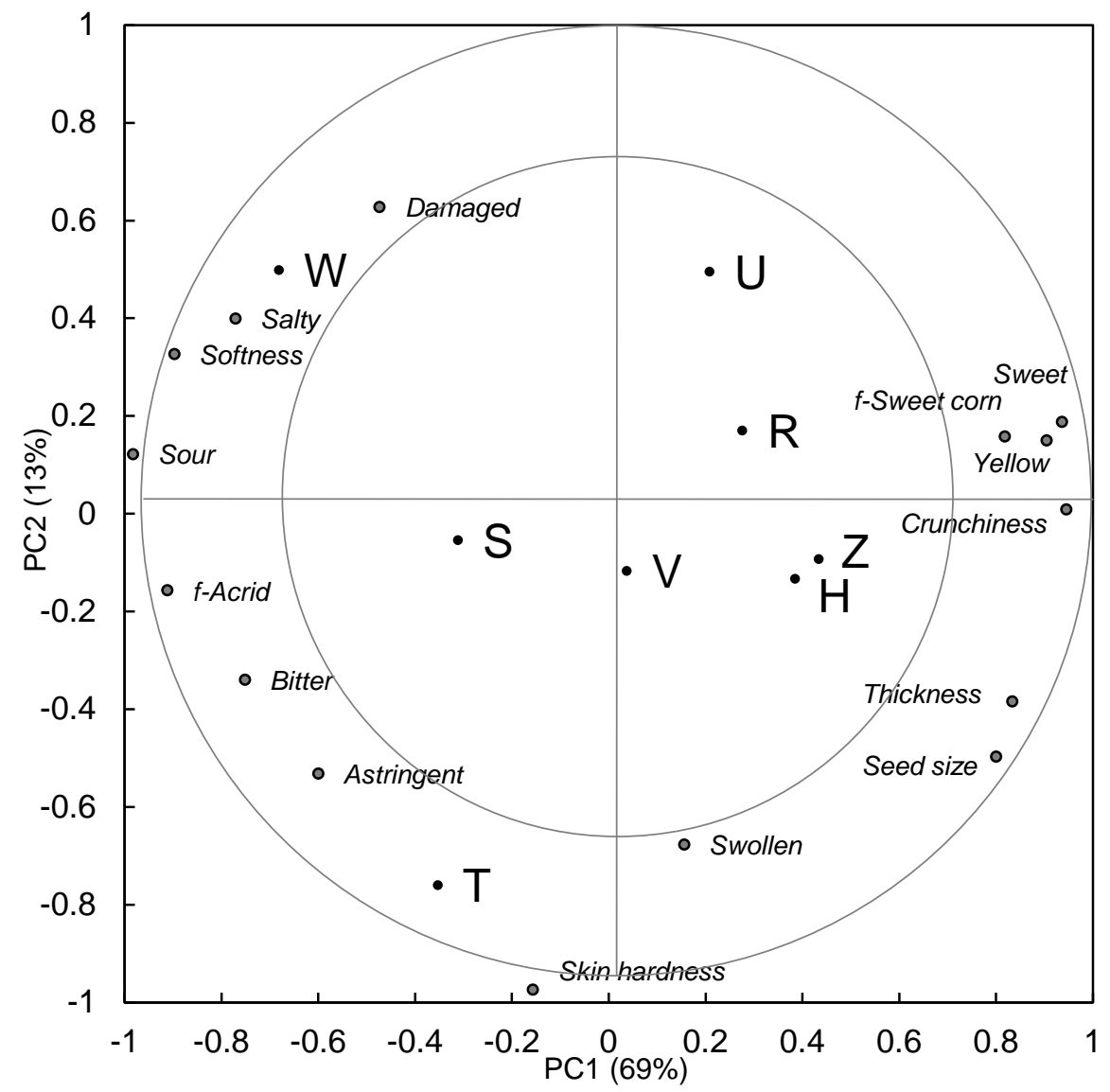



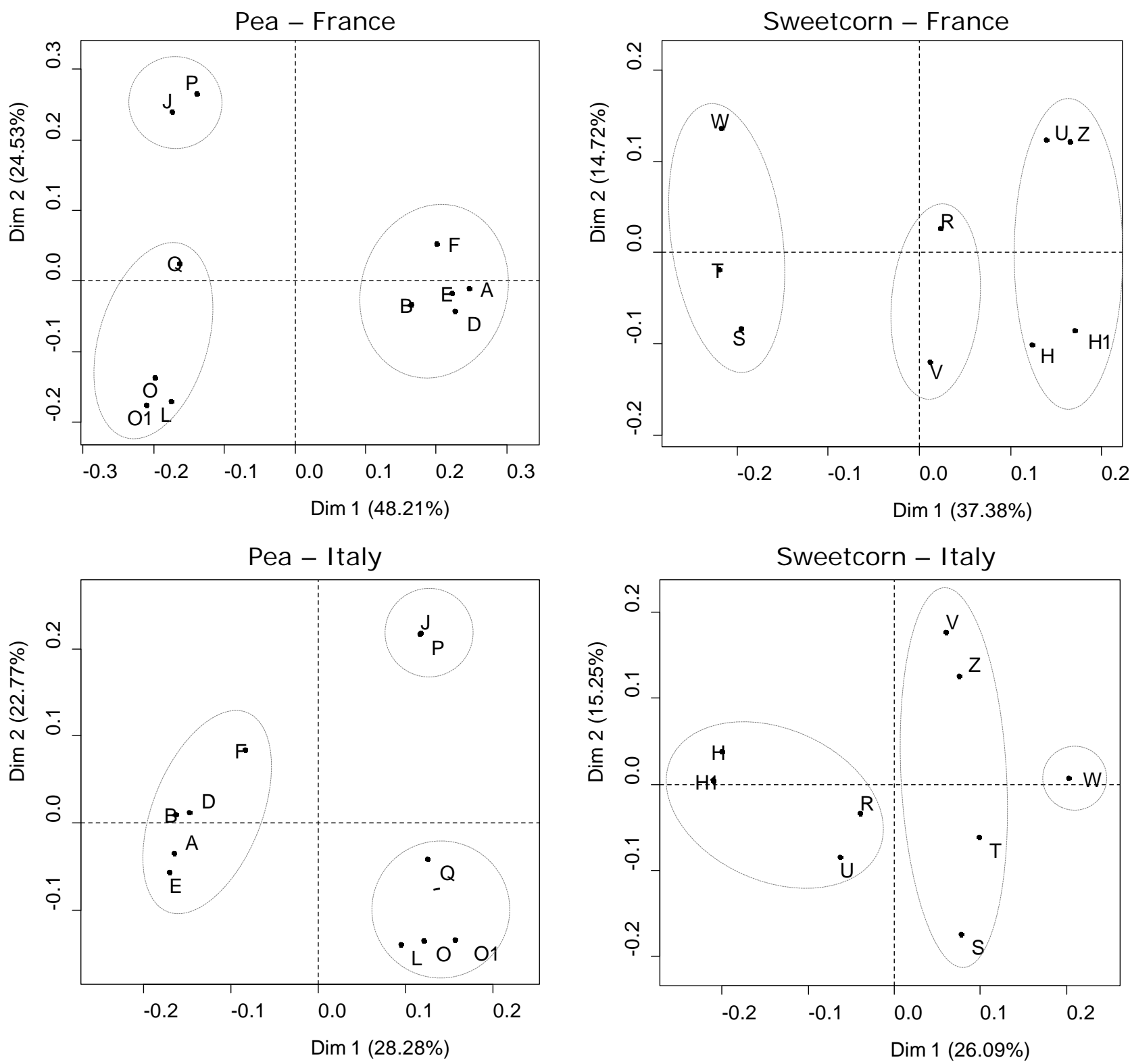

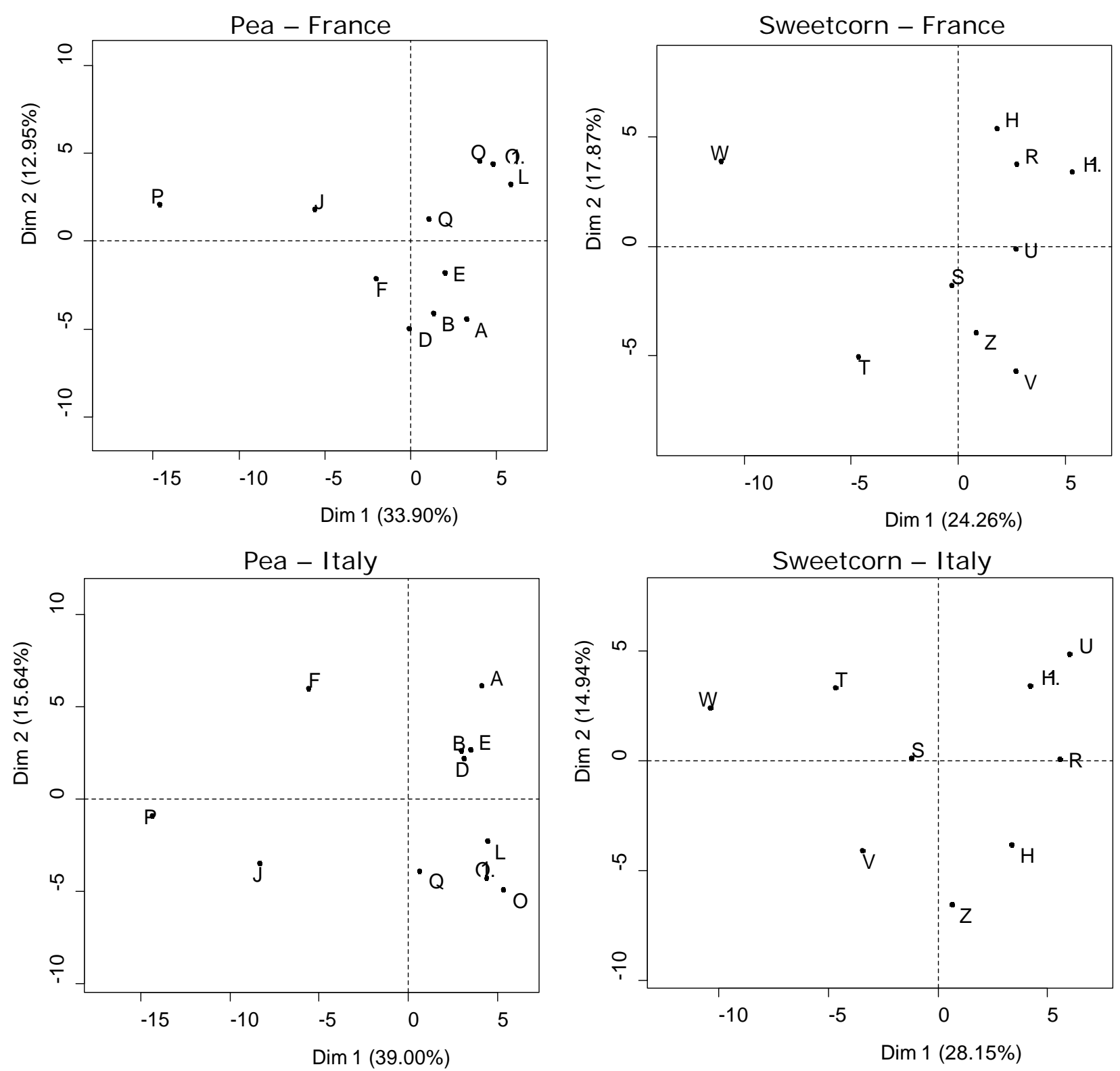

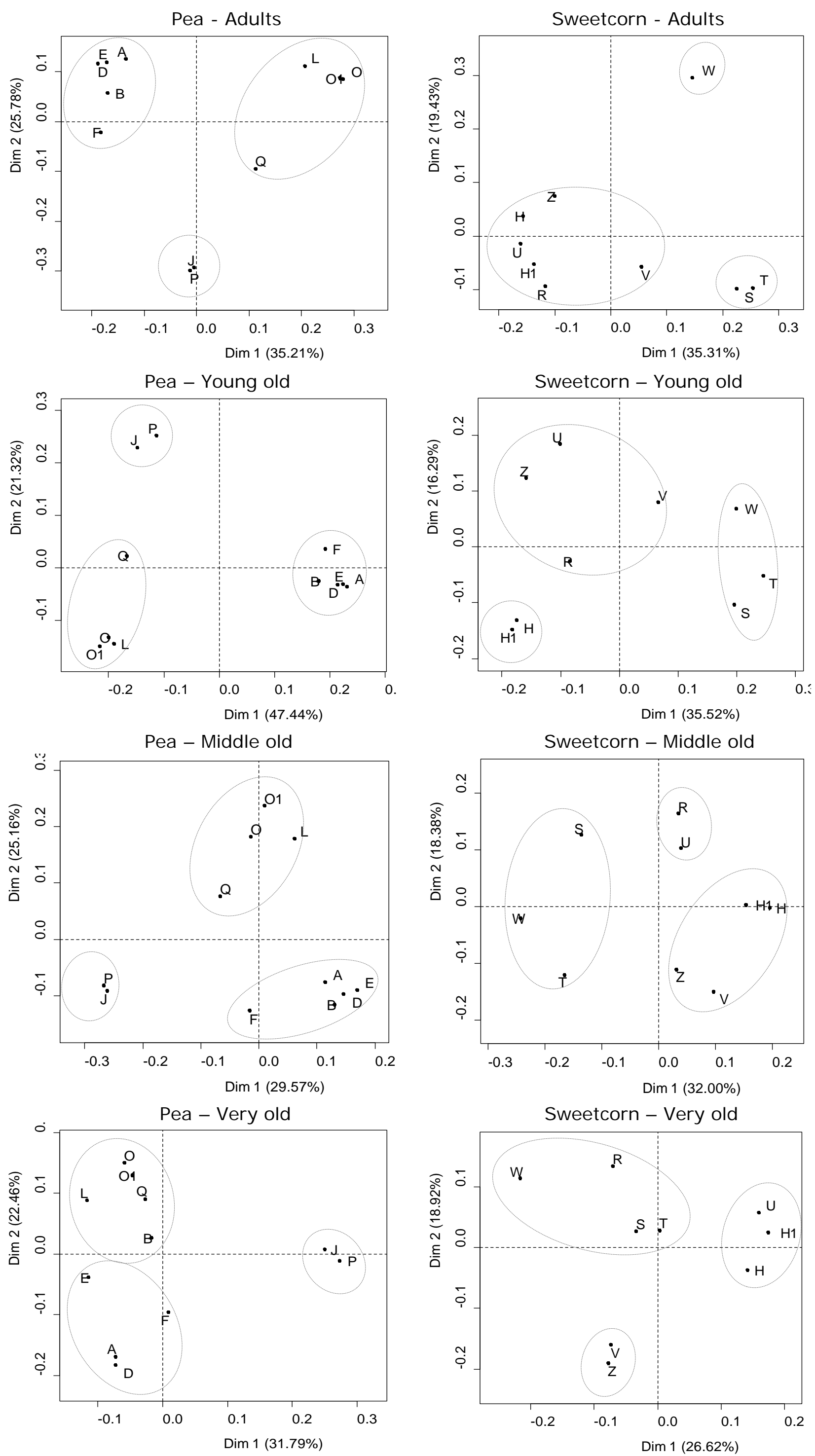

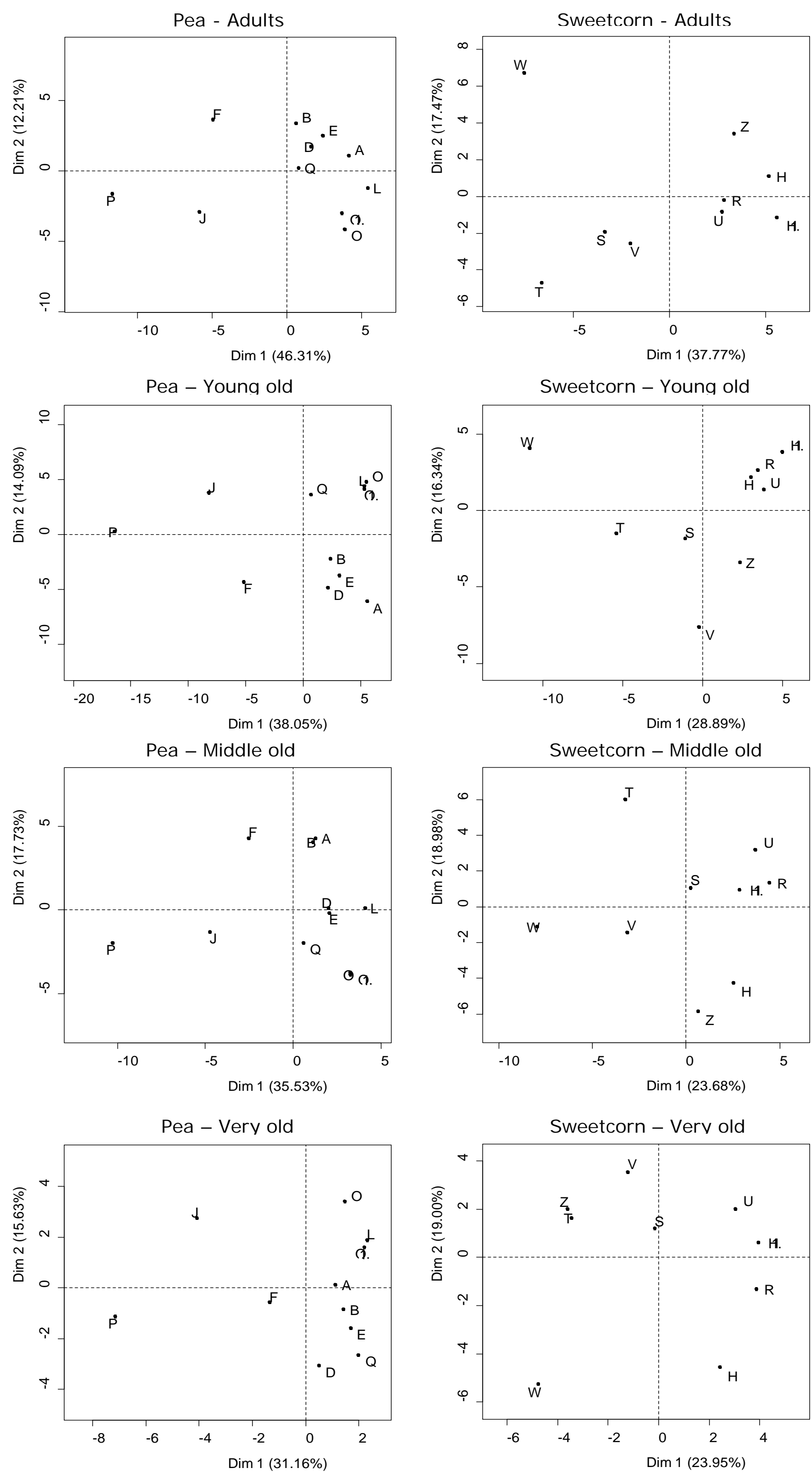
a.

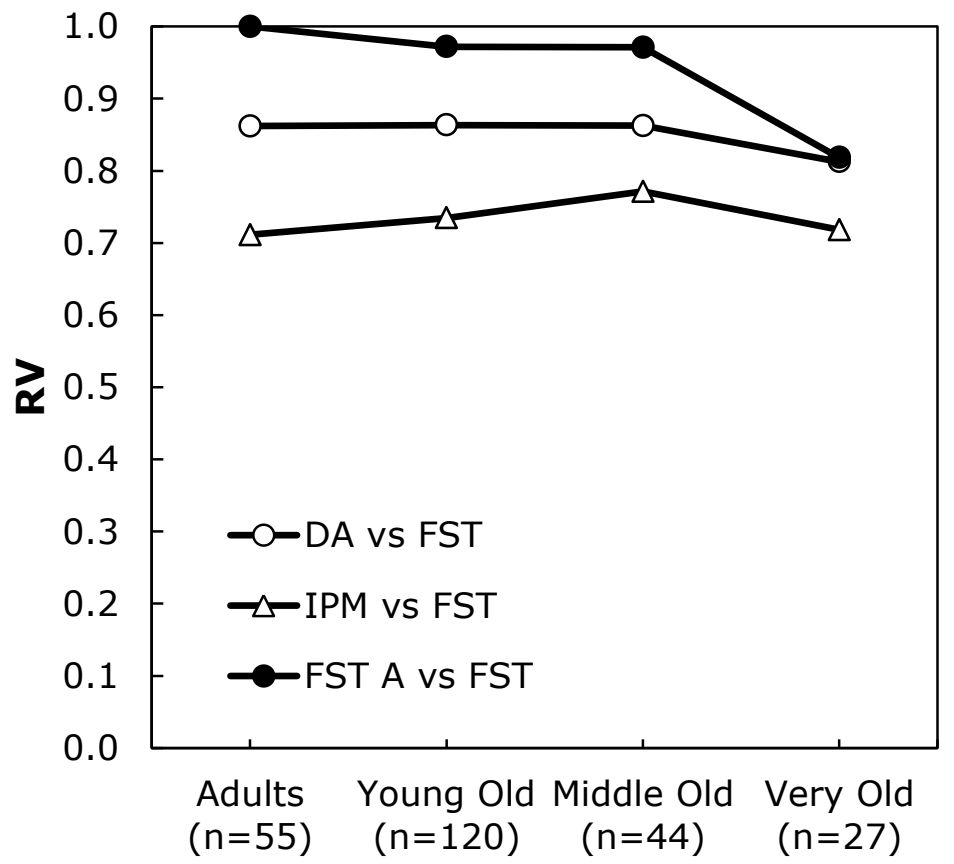

b.

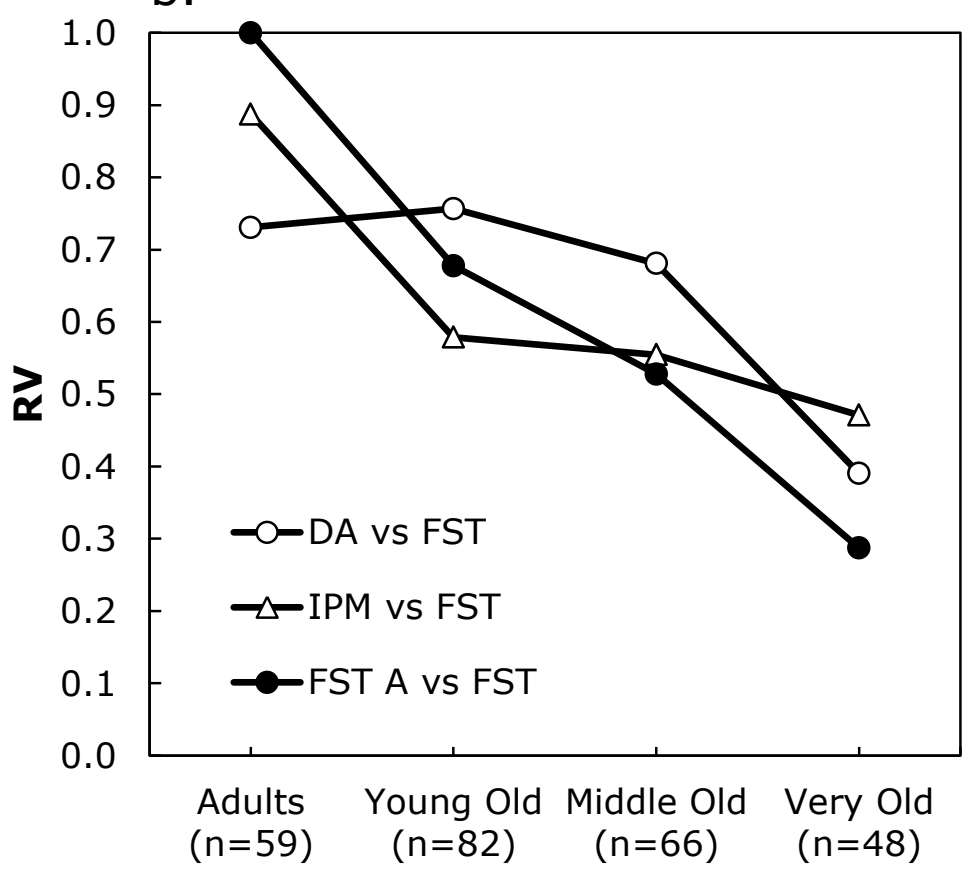


a.

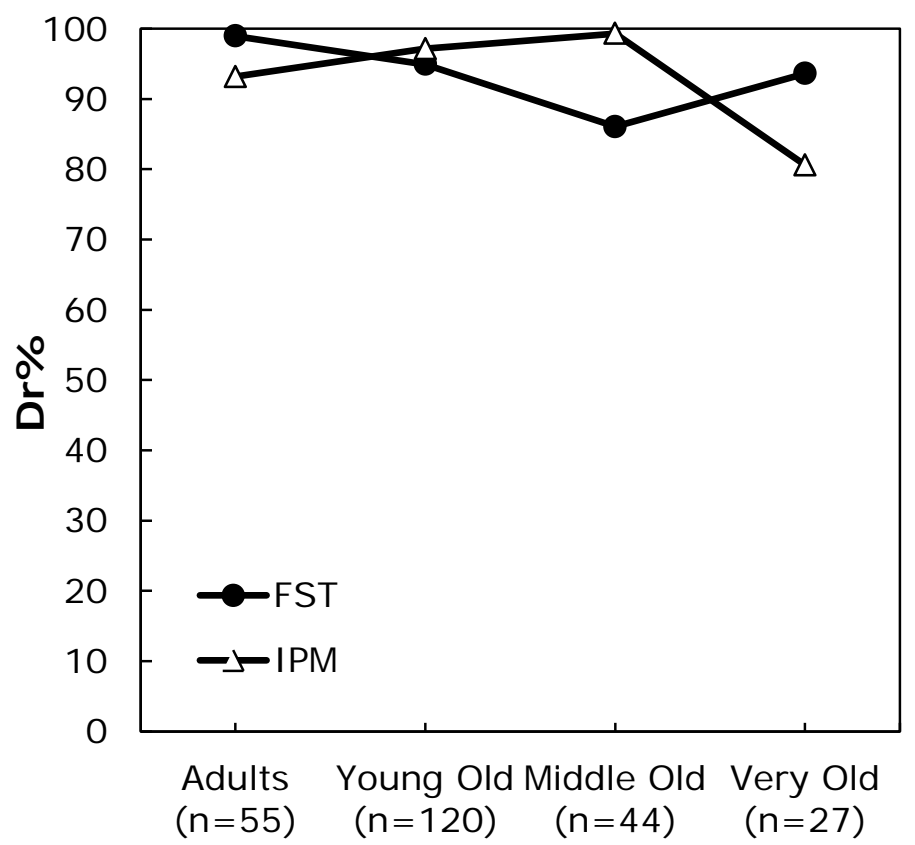

b.

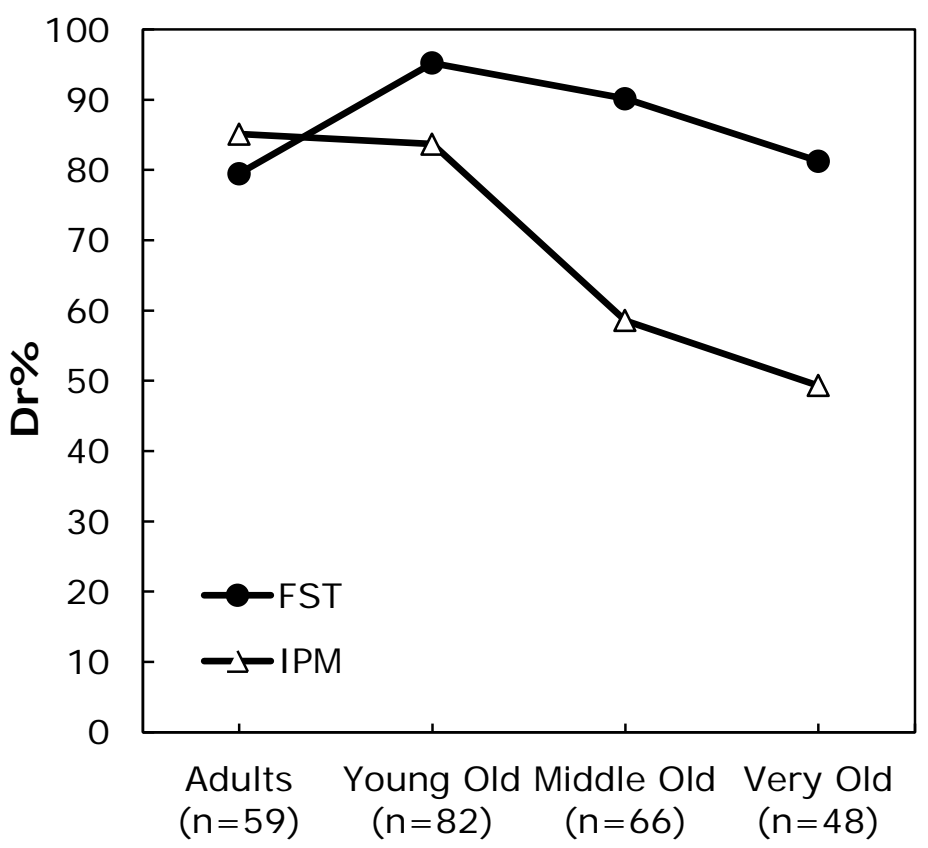

